\title{
Moving breather collisions in Klein-Gordon chains of oscillators
}

\author{
A Alvarez ${ }^{1}$, FR Romero ${ }^{1}, \mathrm{~J} \mathrm{Cuevas}^{2}$, and JFR Archilla ${ }^{2}$ \\ 1 Grupo de Física No Lineal. Área de Física Teórica. Facultad de Física. Universidad de Sevilla. Avda. Reina Mercedes, s/n. \\ 41012-Sevilla (Spain) \\ 2 Grupo de Física No Lineal. Departamento de Fisica Aplicada I. ETSI Informática. Universidad de Sevilla. Avda. Reina \\ Mercedes, s/n. 41012-Sevilla (Spain)
}

Received: date / Revised version: date

\begin{abstract}
We investigate the collisions of moving breathers, with the same frequency, in three different Klein-Gordon chains of oscillators. The on-site potentials are: the asymmetric and soft Morse potential, the symmetric and soft sine-Gordon potential and the symmetric and hard $\phi^{4}$ potential. The simulation of a collision begins generating two identical moving breathers traveling with opposite velocities, they are obtained after perturbing two identical stationary breathers which centers are separated by a fixed number of particles. If this number is odd we obtain an on-site collision, but if this number is even we obtain an inter-site collision. Apart from this distinction, we have considered symmetric collisions, if the colliding moving breathers are vibrating in phase, and anti-symmetric collisions, if the colliding moving breathers are vibrating in anti-phase. The simulations show that the collision properties of the three chains are different. The principal observed phenomena are: breather generation with trapping, with the appearance of two new moving breathers with opposite velocities, and a stationary breather trapped at the collision region; breather generation without trapping, with the appearance of new moving breathers with opposite velocities; breather trapping at the collision region, without the appearance of new moving breathers; and breather reflection. For each Klein-Gordon chain, the collision outcomes depend on the lattice parameters, the frequency of the perturbed stationary breathers, the internal structure of the moving breathers and the number of particles that initially separates the stationary breathers when they are perturbed.
\end{abstract}

PACS. 0 5.45.Yv Solitons - 63.20.Pw Localized modes - 63.20.Ry Anharmonic lattice modes - 66.30.hp Molecular crystals

\section{Introduction}

The study of intrinsic localized modes (ILMs), or discrete breathers (DBs), in lattices of oscillators, is an active research field in nonlinear physics [1-4]. These vibrational modes are rather generic in models of Klein-Gordon and FPU lattices [5-8]. They also appear as solutions of the Discrete Nonlinear Shrödinger (DNLS) equation [9], where these excitations are usually known as discrete solitons.

Under certain conditions, stationary breathers can be set in motion if they experience appropriate perturbations $[10,11]$, and they are called moving breathers (MBs). There are no exact solutions for MBs, but they can be obtained by means of numerical calculations. The conditions for the existence of MBs in Klein-Gordon lattices depend on the exact details of both the on-site and the inter-site potentials. One of the most thoroughly studied KleinGordon models where MBs appear is the Hamiltonian Klein-Gordon chain with Morse on-site potential and harmonic coupling potential [12-14]. Variants of this model

Correspondence to: romero@us.es have been proposed in the study of the DNA molecule, for example the Peyrard-Bishop model $[15,16]$.

Of course, MBs can appear in many other Klein-Gordon models, as in chains with sine-Gordon on-site potential and harmonic coupling potential, or in chains with the $\phi^{4}$ on-site potential.

In a real discrete system, MBs should appear at arbitrary positions, then, it is natural to be interested in their collisions. The study of collisions of MBs in FPU chains was initiated in [17]. However, in Klein-Gordon chains, the studies were initially limited to the interaction of moving low-amplitude breathers with stationary high-amplitude ones [18-20], or to the interaction between quasi-periodic moving breathers in dissipative lattices [21]. More recently, some results have been obtained considering head-on collisions in a Klein-Gordon chain with the Morse potential and harmonic coupling potential [32].

The study of soliton collisions in non-integrable DNLS models has been undertaken very recently. These models deal with nearly integrable DNLS equations [22,23], cubic DNLS equations [24] and saturable DNLS equations [25, $26]$. 
The aim of this paper is to get some insight into the detailed mechanisms and possible outcomes of the collisions in three different Klein-Gordon chains of oscillators. The on-site potentials are: the soft Morse potential, the soft sine-Gordon potential and the hard $\phi^{4}$ potential.

This article is organized as follows. Sec. 2 introduces the models, describes the technics for generating MBs and the different types of collisions. Sec. 3, presents the numerical simulations results corresponding to symmetric and anti-symmetric collisions in Morse chains. Sec. 4 presents the outcomes corresponding to the same type of collisions in sine-Gordon chains. Sec. 5 is dedicated to the collisions in $\phi^{4}$ chains. The stability analysis of the stationary breathers makes possible to get some insights into the trapping phenomena that appear after some collisions, an approach is presented in Sec. 6. Finally, the conclusions are presented in Sec. 7 .

\section{Moving breathers in Klein-Gordon chains}

In scaled variables, the Hamiltonian of a Klein-Gordon chain of identical oscillators with nearest-neighbor interacting potential is given by:

$$
H=\sum_{n}\left[\frac{1}{2} \dot{u}_{n}^{2}+V\left(u_{n}\right)+U\left(u_{n}-u_{n-1}\right)\right]
$$

where $u_{n}$ represents the displacement of the $n^{\text {th }}$ oscillator from the equilibrium position, $V\left(u_{n}\right)$ is the on-site potential and $U\left(u_{n}-u_{n-1}\right)$ is the inter-site potential.

In this paper we have considered three different KleinGordon chains with the following Hamiltonians

\section{Morse chain:}

$$
H=\sum_{n}\left[\frac{1}{2} \dot{u}_{n}^{2}+\frac{1}{2}\left(\exp \left(-u_{n}\right)-1\right)^{2}+\frac{1}{2} \varepsilon\left(u_{n}-u_{n-1}\right)^{2}\right]
$$

2. sine-Gordon chain:

$$
H=\sum_{n}\left[\frac{1}{2} \dot{u}_{n}^{2}+\frac{1-\cos 2 \pi u_{n}}{4 \pi^{2}}+\frac{1}{2} \varepsilon\left(u_{n}-u_{n-1}\right)^{2}\right]
$$

3. $\phi^{4}$ chain:

$$
H=\sum_{n}\left[\frac{1}{2} \dot{u}_{n}^{2}+\frac{u_{n}^{2}}{2}+\frac{u_{n}^{4}}{4}+\varepsilon V\left(u_{n}-u_{n-1}\right)\right]
$$

with $V\left(u_{n}\right)=\frac{u_{n}^{2}}{2}+\frac{u_{n}^{4}}{4}$.

The on-site potentials of these chains are, respectively, the asymmetric soft Morse potential, the symmetric soft sine-Gordon potential and the symmetric hard $\phi^{4}$ potential. In all cases $\varepsilon$ is a coupling parameter. Notice that the inter-site potential of the $\phi^{4}$ chain is non harmonic, while the other two chains have harmonic potentials.

Time-reversible, stationary breathers can be obtained using methods based on the anti-continuous limit [27]. Initially, $\dot{u}_{n}=0, \forall n$, and the displacements of a stationary breather centered at $n_{0}$ are denoted by $\left\{u_{S B, n}\right\}$. A moving breather $\left\{u_{t, n}\right\}$ can be obtained with an appropriate perturbation of the stationary breather. One possibility consists in modifying its initial conditions as follows:

$$
\begin{aligned}
& u_{M B, n}^{0}=u_{S B, n} \cos \left(\alpha\left(n-n_{0}\right)\right) \\
& \dot{u}_{M B, n}^{0}= \pm u_{S B, n} \sin \left(\alpha\left(n-n_{0}\right)\right) .
\end{aligned}
$$

The plus-sign gives rise to breathers traveling towards the positive direction, and the minus-sign towards the opposite one. This procedure, taken from the DNLS context $[24,25]$, works as good as the marginal-mode method [10, $11]$ and gives good mobility for a large range of $\varepsilon$ values. We take Eqs. (5) as the initial conditions for integrating the dynamical equations using a symplectic algorithm [28].

The characteristics and internal structure of a MB depend on the potentials $V\left(u_{n}\right)$ and $U\left(u_{n}-u_{n-1}\right)$, also on the specific form of the perturbation given to the stationary breather. In our case, the parameter $\alpha$ is involved in this perturbation, it represents the difference of phase between two neighboring oscillators and we will refer to it as the wave number. We have proved that the translational velocity and the translational kinetic energy of the MB increase with $\alpha$.

The study begins generating two identical stationary breathers, with the same frequency, separated by a given number of particles between their centers. Both breathers are in phase, that is, before the perturbation each breather is always like the mirror image of the other one. For obtaining symmetric collisions, the perturbation should be given simultaneously to both breathers using the initial conditions Eqs. (5), with the plus sign for one breather and the minus sign for the other one. In this way the MBs travel with the same modulus of velocity, but opposite directions, and they are in phase.

The DBs can be perturbed at different instants of time and then the MBs are out of phase. It is possible, for example, to have the MBs vibrating in anti-phase, i.e, with a phase difference $\pi$. We call to these collisions antisymmetric collisions.

The MBs in the three chains have very different spatial profiles. Fig. 1 shows the spatial profiles of the MBs in the three chains at different instants of time.

We call $N_{c}$ the number of particles separating initially the centers of the two DBs, and we distinguish two types of collisions: a) on-site collisions (OS), if $N_{c}$ is an odd number, that is, the middle of the initial separation coincides with a particle; and b) inter-site collisions (IS), if $N_{c}$ is even $[24,25]$.

\section{Collision simulations in Morse chains}

In a previous paper [32] some results were obtained considering symmetric collisions in a Klein-Gordon chain with 

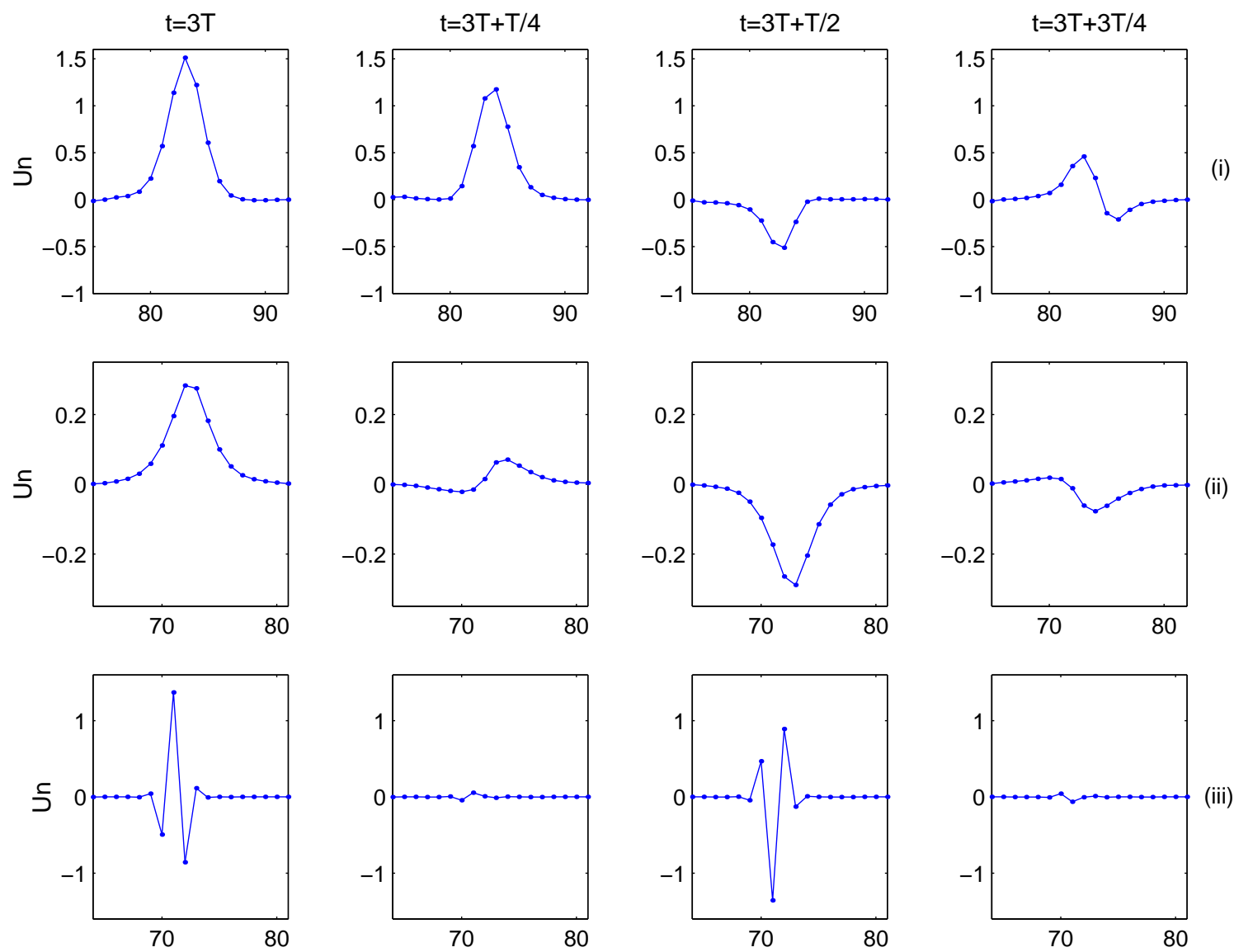

Fig. 1. Spatial profiles of the MBs at different instants of time. (i) Morse chain; (ii) sine-Gordon chain; (iii) $\phi^{4}$ chain. The perturbation is given at $t=0$ and for each case T represents the period of the respective DB. Parameters: Morse chain $\left(\omega_{\mathrm{b}}=0.8\right.$, $\varepsilon=0.32, \alpha=0.138)$; sine-Gordon chain $\left(\omega_{\mathrm{b}}=0.9, \varepsilon=0.5, \alpha=0.138\right) ; \phi^{4}$ chain $\left(\omega_{\mathrm{b}}=3, \varepsilon=0.53, \alpha=0.138\right)$

Morse on-site potential and harmonic coupling potential. We considered different symmetric collisions varying the parameter $\alpha$ and maintaining fixed the number $N_{c}$, thus the colliding entities change for each value of $\alpha$, and we analyzed the possible outcomes.

In this paper we have taken a different approach, we analyze different symmetric, and also anti-symmetric collisions, with a fixed value of $\alpha$ and different values of $N_{c}$, in this way the colliding MBs are always identical . When $N_{c}$ changes, the dynamical states of the MBs changes when the collision begins. Thus, this approach is complementary to the previous one.

We write

$$
N_{c}=N_{o}+j j
$$

where $N_{o}$ is a fixed number to guarantee that the breathers are initially far apart, and jj is a positive even number. Thus, for OS collisions $N_{o}$ is odd, and for IS collisions $N_{o}$ is even.

Although the MBs are identical when $j j$ varies, the time passed between the initial perturbation and the initiation of the collision increases with $j j$. The possible different outcomes should be due to the different internal states of motion of the MBs when they collide.
The breather frequency $\omega_{\mathrm{b}}$ is below the phonon band because the Morse on-site potential is soft. The lowest frequency of the phonon band is equal to the linear frequency of an isolated oscillator and is given by $\omega_{0}=V^{\prime \prime}(0)^{1 / 2}$. For breathers with small amplitude the system is close to the linear limit, and their frequency is close to $\omega_{0}$. Therefore, $\left|\omega_{0}-\omega_{\mathrm{b}}\right|$ is a measure of how far the system is from the linear regime. Hence, it is natural to think that the collision scenario should be strongly dependent of the common frequency of the two MBs. For that reason in [32] we considered two different breather frequencies, $\omega_{\mathrm{b}}=0.8$ and $\omega_{\mathrm{b}}=0.95$, that represent different degrees of nonlinearity. In this paper we consider only the frequency $\omega_{\mathrm{b}}=0.8$ which represents an intermediate degree of nonlinearity.

Before considering collisions between MBs, it is necessary to have an estimate of their lifetime. A MB has not a single frequency but a continuous band around the frequency of the stationary breather. As this band contains phonon frequencies, phonons are excited and the MB loses energy. However, we have checked that in our systems this energy is lost at a very small rate and MBs propagate during several hundreds of periods without apparent decay. In our simulations, the time before the collision is between 
20 and 80 periods, therefore, we can consider that the colliding breathers are almost intact.

\subsection{Moving breather collisions with fixed $N_{c}$ and different values of $\alpha$}

The results relative to OS symmetric collisions presented in [32] show that there exists a great sensitivity of the outcomes with respect to the parameter $\alpha$. Fig. 2 shows MB collisions corresponding to a frequency $\omega_{\mathrm{b}}=0.8$, coupling parameter $\varepsilon=0.32, N_{c}=41$ and three $\alpha$ values that are representative of the different scenarios that can be found. They can be summarized as follow:

\section{MB generation with trapping:}

The collision produces new breathers, a trapped one located at the collision region, and two new symmetric MBs, as Fig. 2(a) shows for $\alpha=0.048$. The trapped breather contains most of the initial energy. This behavior has been described in the pioneering work cited in Ref.[10].

Varying the parameter $\alpha$, it is possible to obtain a noticeable attenuation of the amplitude of the trapped breather as it is shown in Fig. 2(b) which corresponds to $\alpha=0.18$. In this case the emerging MBs contain most of the initial energy.

2. MB generation without trapping:

With a slightly different value of $\alpha$, it is possible a collision which results in two new symmetric MBs, with almost the same velocity that the colliding breathers'. This is shown in Fig. 2(c) for $\alpha=0.19$.

The total energy transported by the colliding MBs is distributed after the collision: some part corresponds to the energy of the trapped breather, another part to the emerging MBs, and a small fraction of the energy is transferred to the lattice in the form of phonon radiation. In order to illustrate this phenomenon, we have studied the evolution of the "central energy", defined in our study as the energy of eleven particles around the collision region. This number of particles has been selected because it corresponds to the typical size of a discrete breather with the parameters used. Fig. 3 shows the evolution of the central energy for the three cases considered in Fig. 2.

Before the collision the central energy is zero; after the initiation of the collision it increases quickly, up to a value very close to the sum of the incident MB energies; the subsequent decrease of the central energy is caused by the appearance of new emerging MBs (some of them of short life) and by the phonon radiation.

For each set of values $(\varepsilon, \alpha)$, we can calculate the relative trapped energy after the collision, defined as the ratio between the energy of the trapped breather and the sum of the energies of the two incident MBs. Taking $\varepsilon=0.32$ and $\alpha \in[0.030,0.200]$ with step size 0.002 , we have calculated the relative trapped energies of these collisions which are represented by the distribution of points shown in Fig. 4 (left).

Notice that almost all the points are distributed inside a band apparently at random. This means that a small change of $\alpha$ can affects the amount of energy of the trapped breather. Occasionally, for large enough values of $\alpha$, there are points outside the band corresponding to a relative trapped energy close to zero, i.e., for those values of $\alpha$, two new MBs are generated without trapping. The sensitivity with respect to $\alpha$ can be better appreciated if we choose a smaller step size for $\alpha$. If we take $\alpha$ within the interval $[0.1360,0.1400]$ with step size 0.0002 , we find, for example, MB generation with trapping for $\alpha=0.1372$ and MB generation without trapping for $\alpha=0.1374$ (see Fig. 4 (right)).

Generally, the simulations show that the outcome is strongly sensitive to the exact dynamical states of the MBs just before the collision. MBs with different values of $\alpha$ have different velocities, thus when the collision begins they have different internal states of motion.

The Fourier spectra of the breathers involved in the collisions of Fig. 2(a) are shown in Fig. 5. The trapped breather has a frequency $\left(\omega_{\text {trap }} \sim 0.77\right)$, which is lower than the colliding breathers', but its amplitude is larger. The emerging MBs have higher frequency $\left(\omega_{\mathrm{e}} \sim 0.90\right)$ and smaller amplitude than the colliding breathers'. We have also obtained the Fourier spectra for the breathers shown in Fig. 2(b) and (c). For the former, the trapped and emerging breathers have frequencies higher than the incident ones' ( $\omega_{\text {trap }}=0.90$ and $\omega_{\mathrm{e}}=0.86$, respectively), but smaller amplitudes. For the latter, the frequency of the generated breathers is the same as the incident ones' indicating in this case the quasi-elastic character of the scattering.

Although the quantitative changes are not significant, they give an indication of the type of scattering produced.

We have also performed an extensive study of collisions considering different values of the coupling parameter $\varepsilon$ and MBs with different values of the wave number $\alpha$. The values of $\varepsilon$ have been taken in the interval $[0.13,0.35]$ with step size 0.01 . For each value of $\varepsilon$ the values of $\alpha$ have been taken in the interval $[0.030,0.200]$ with step size 0.002 . We observe that the distributions of points are similar although the mean values of the relative trapped energies and the dispersion of points change, and we refer to [32] to see the quantitative changes.

We have observed that there are not significant qualitative differences between the OS and IS collisions.

\subsection{Moving breather collisions with fixed $\alpha$ and different values of $N_{c}$}

In the previous simulations all the MBs are obtained perturbing two identical DBs with their centers separated by a fixed number of particles $N_{c}$. The velocity of a MB depends on the parameter $\alpha$, thus the comparison of the outcomes always refers to collisions of MBs with different characteristic. Another approach is to fix the parameter $\alpha$ and perturb the DBs varying their separation $N_{c}$, thus the only difference between two collisions is the time passed between the initial perturbation and the initiation of the collision. We describe separately the symmetric and the 
(a) $\alpha=0.048$

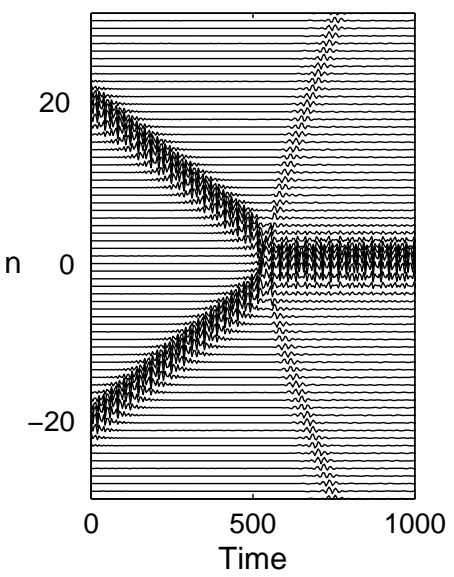

(b) $\alpha=0.18$

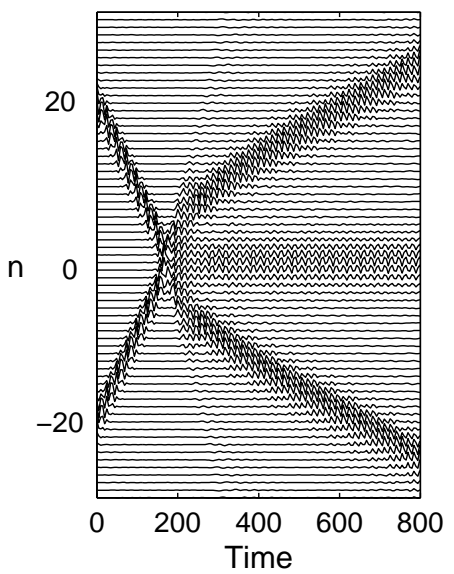

(c) $\alpha=0.19$

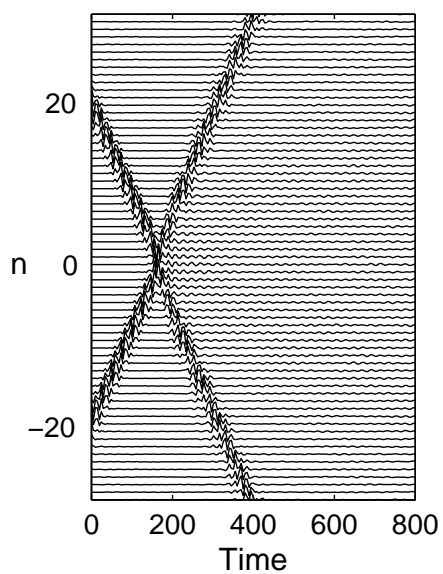

Fig. 2. Three examples of symmetric collisions with coupling parameter $\varepsilon=0.32$ and frequency $\omega_{\mathrm{b}}=0.8$. Displacements versus time for three different values of the wave number $\alpha$ : (a) $\alpha=0.048$; (b) $\alpha=0.18$; (c) $\alpha=0.19$. Notice that these behaviors occur in an apparently random way when $\alpha$ increases, although in these figures they seem to take place progressively.

(a) $\alpha=0.048$

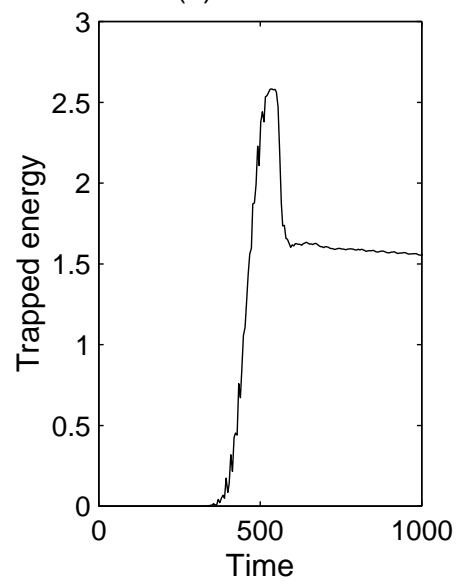

(b) $\alpha=0.18$

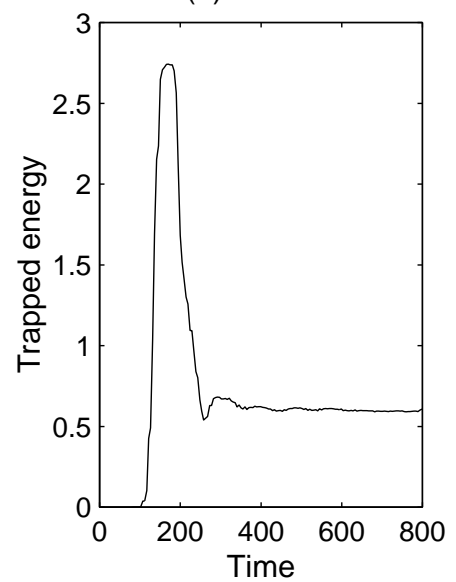

(c) $\alpha=0.19$

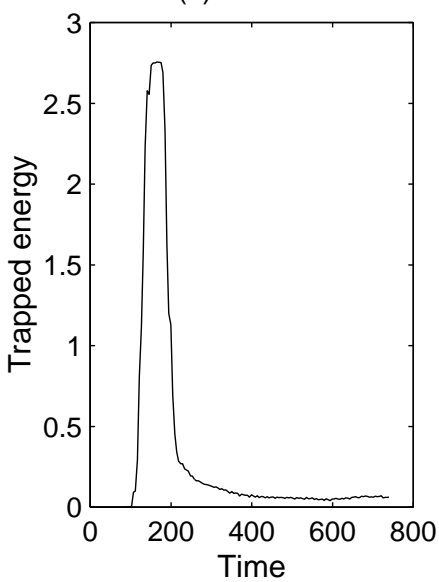

Fig. 3. Time evolution of the central energy corresponding to the collisions (a), (b) and (c) of Fig. 2, respectively.
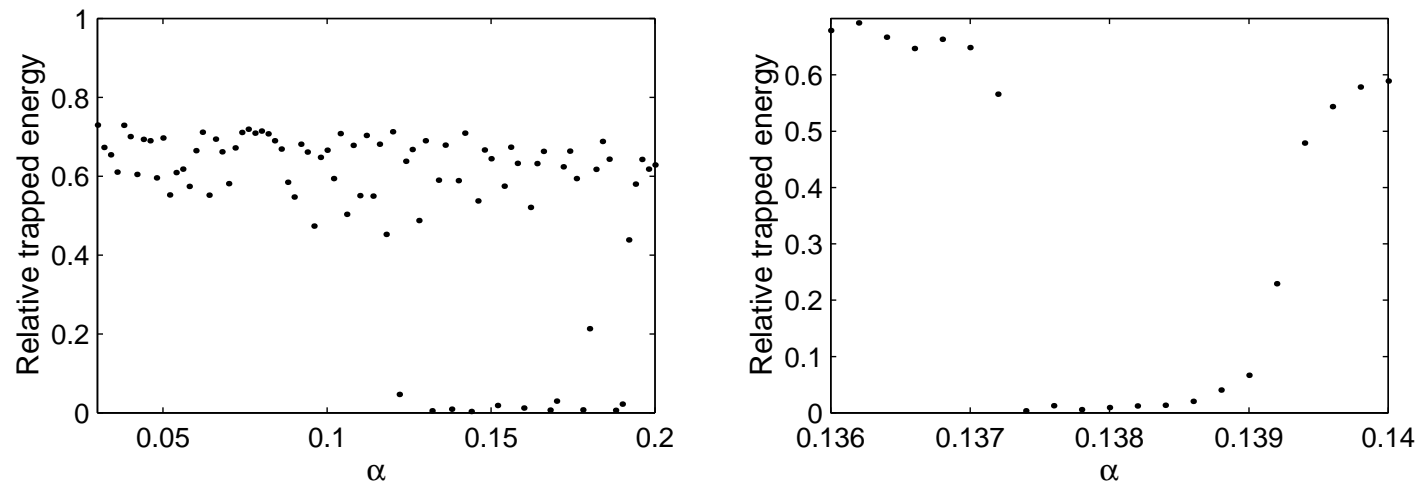

Fig. 4. (Left) Distribution of points representing the relative trapped energy versus $\alpha, \alpha \in[0.030,0.200]$ with step size 0.002 . Coupling parameter $\varepsilon=0.32$ and breather frequency $\omega_{\mathrm{b}}=0.8$. (Right) Zoom around a value corresponding to MB generation without trapping. 
(a)

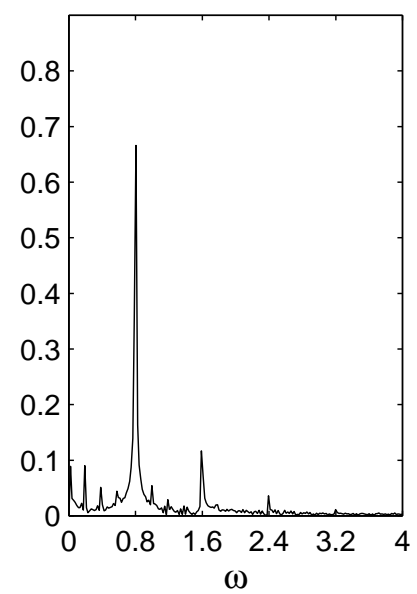

(b)

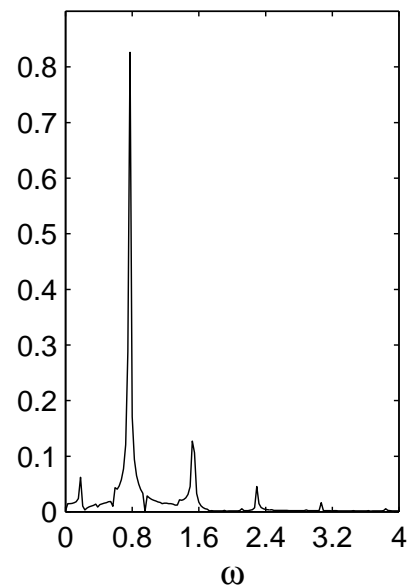

(c)

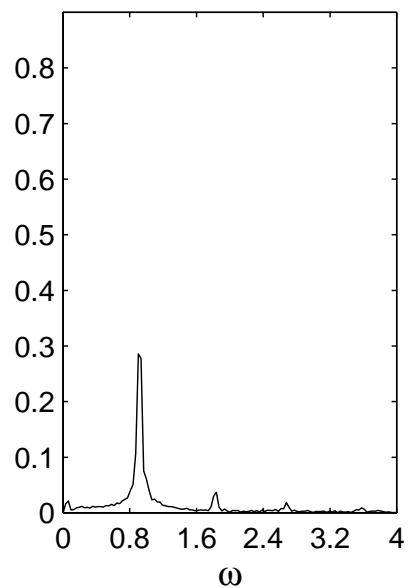

Fig. 5. Fourier spectra of the breathers involved in the collisions shown in Fig. 2(a). a) Incident breathers; b) trapped breather; c) emerging breathers.

anti-symmetric collisions, and for each case we consider both OS and IS collisions.

\subsubsection{Symmetric collisions}

First we consider OS symmetric collisions, that is, $N_{c}$ is always odd and the DBs are perturbed simultaneously. We have taken $N_{o}=41$ and $j j$ varies in the interval $[0,100]$ with step size 2 . Then, up to fifty different collisions can be analyzed for a fixed value of $\alpha$.

We have performed an extensive study of symmetric collisions considering different values of the coupling parameter $\varepsilon$ and MBs with different values of $\alpha$. The values of $\varepsilon$ have been taken in the interval $[0.13,0.35]$ and for each value of $\varepsilon$ the values of $\alpha$ have been taken in the interval $[0.030,0.200]$. We present the results obtained with $\varepsilon=0.32$ and $\alpha=0.048, \alpha=0.138$, and $\alpha=0.18$, which correspond to MBs with increasing velocities. These $\alpha$ values are representative of the different scenarios that can be found. Fig. 6 represents the trapped energy versus $j j$ for these three cases.

Fig. 6(left) corresponds to a case with small velocity, i.e., $\alpha=0.048$, the distribution of points appears in a narrow band and there are no points with trapped energy close to zero. When the MBs have small enough kinetic energy, the collision produces MB generation with trapping and most of the energy gets trapped after the collision.

For bigger values of $\alpha$ the phenomenon of MB generation without trapping, appears for some values of $j j$. This can be appreciated in Fig. 6(central), obtained with $\alpha=0.138$, where some points appear with trapped energy close to zero, this means that after the collision almost all the energy is transported by two emerging MBs traveling in opposite directions. For this value of $\alpha$ two consecutive points in the upper band are followed by one point that fall down close to zero.
For $\alpha=0.18$, several consecutive points in the upper band are followed by several consecutive points close to zero. This means that there are some successive values of $j j$ associated with MB generation with trapping, followed by other ones associated with MB generation without trapping, see Fig. 6(rigth). For greater values of $\alpha$ the size of the alternating intervals increases. For example, for $\alpha=0,19$ and $j j \in[0,200]$ a lower band appears in the interval $[0,100]$ and an upper band in the interval $[102,200]$.

Fig. 7 shows the displacements versus time for eight collisions of Fig. 6(central), corresponding to $j j \in[34,48]$, with the fixed value $\alpha=0.138$, coupling parameter $\varepsilon=$ 0.32 and breather frequency $\omega_{\mathrm{b}}=0.8$. The collision outcome is very sensitive to the exact dynamical state when the collision begins. The final state of the collision process can change drastically for two consecutive values of $j j$ maintaining the rest of the parameters unchanged. This can be better appreciate in Fig. 8 that shows a zoom of the collision region corresponding to the pictures with $j j=38$ and $j j=40$ of Fig. 7 .

Fig. 9 shows the evolution of the trapped energy corresponding to four collisions of Fig. 7. For the cases with $j j=36$ and $j j=38$, most of the energy remains trapped after the collision, whereas for the cases with $j j=34$ and $j j=40$, the trapped energy is close to zero.

The qualitative results are similar for other values of $(\varepsilon, \alpha)$. For a given value of the coupling parameter $\varepsilon$, MB generation without trapping begins to appear for $\alpha$ over a certain value. This value increases when the coupling parameter $\varepsilon$ decreases and with a small value of $\varepsilon$, for example with $\varepsilon=0.15$, there is always trapping with $\mathrm{MB}$ generation.

We have observed that there are no significant qualitative differences between the outcomes of OS and IS collisions.

The results of subsections 3.1 and 3.2 show that, for a given values of $\varepsilon$ and $\omega_{\mathrm{b}}$, the relevant parameters to 

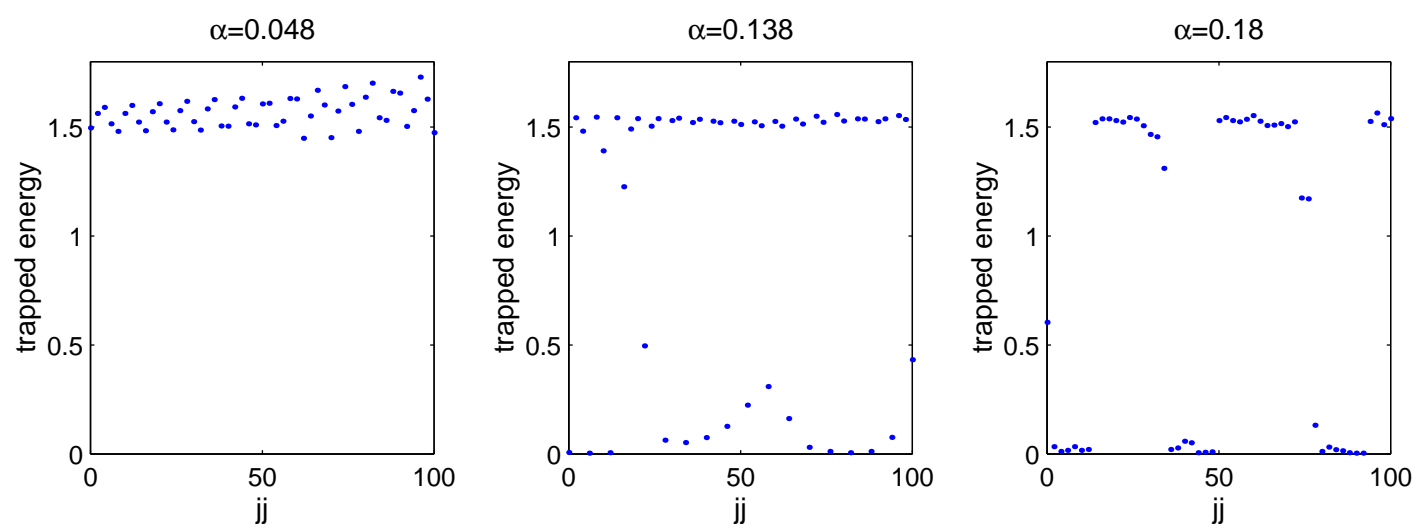

Fig. 6. Three distributions of points representing the trapped energy versus $j j$, for $\alpha=0.048, \alpha=0.138$, and $\alpha=0.18$, respectively. Coupling parameter $\varepsilon=0.32$ and breather frequency $\omega_{\mathrm{b}}=0.8$.
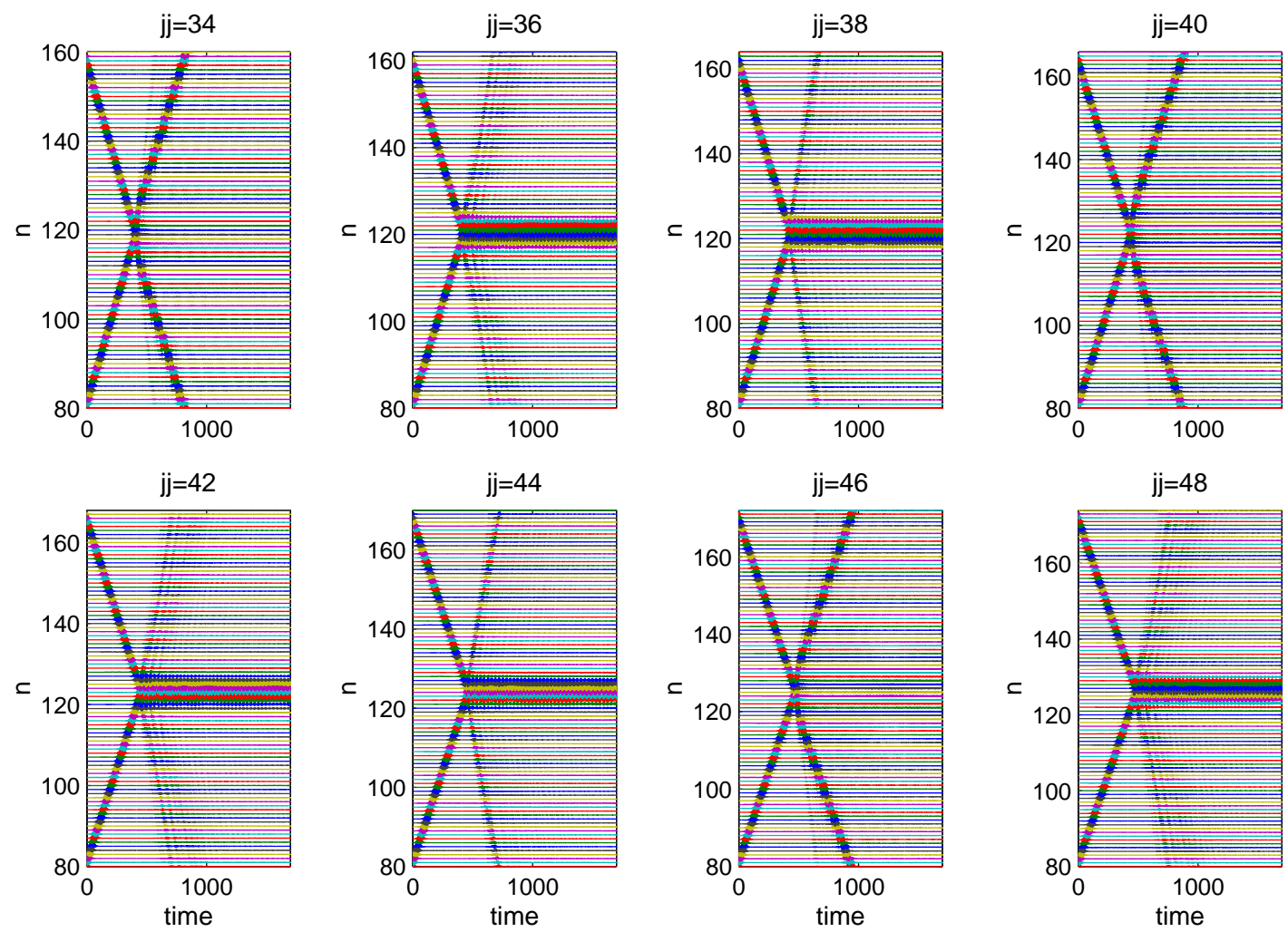

Fig. 7. Morse chain. Displacements versus time for eight OS symmetric collisions corresponding to $j j \in[34,48]$, with the fixed value $\alpha=0.138$. Coupling parameter $\varepsilon=0.32$ and breather frequency $\omega_{\mathrm{b}}=0.8$.

determine the outcomes of the collisions are both $\alpha$ and the number $N_{c}$.

\subsubsection{Anti-symmetric collisions}

For both OS and IS anti-symmetric collisions the MBs are always reflected, each MB rebounds after the collision with the same but opposite velocity. Now we will distinguish between $\mathrm{MB}$ generation (without trapping or with trapping) and $\mathrm{MB}$ reflection.
In the first case, there is a positive superposition of the vibration of particles at the collision region. After the collision new MBs can emerge with the same or different velocities that the incoming breathers' (usually, with a slightly different frequency) and eventually a trap breather appears.

In the case of $\mathrm{MB}$ reflection, either one central particle (OS collisions) or two central particles (IS collisions) remain at rest or nearly at rest at the collision region and the incoming breathers simply interchange the direction of their velocities. Fig. 10(left) shows a zoom of the colli- 

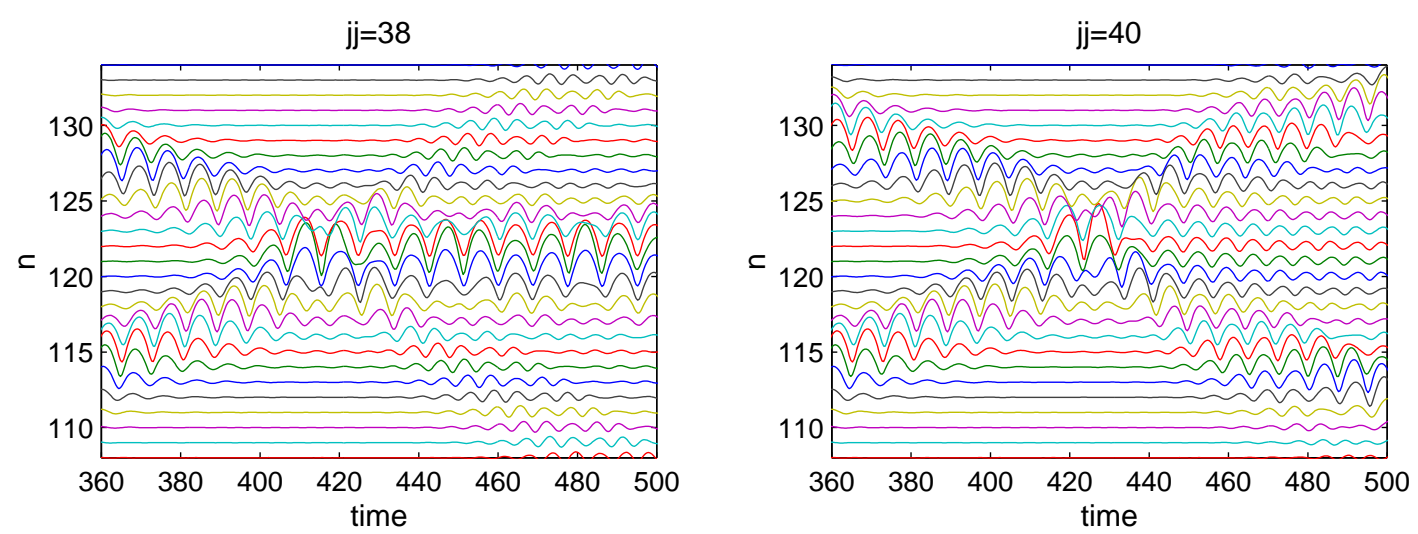

Fig. 8. Morse chain. Zoom of the collision region corresponding to two cases of Fig. 7: (Left) MB generation with trapping with $\mathrm{jj}=38$. (Right) MB generation without trapping with $\mathrm{jj}=40$.
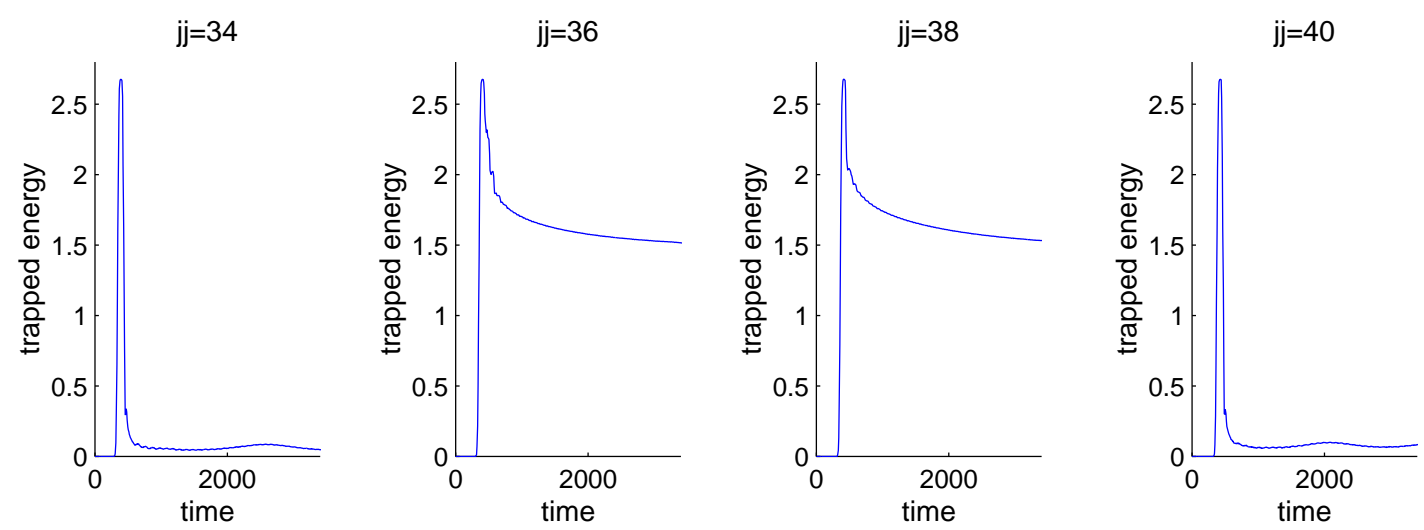

Fig. 9. Morse chain. Trapped energy versus time corresponding to the first four collisions of Fig. 7, respectively.

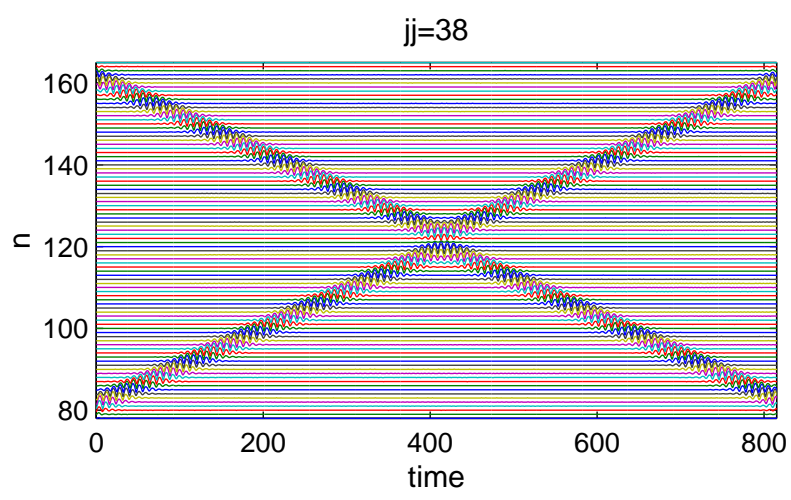

Fig. 11. Morse chain. Displacements versus time of an OS antisymmetric collision with $j j=38$ and the same parameters of Fig. 7

sion region corresponding to a case of $\mathrm{MB}$ reflection and Fig. 10(right) shows a zoom of a MB generation without trapping.

Fig. 11 shows an OS anti-symmetric collision with $j j=$ 38. Notice the difference of this collision with the third symmetric collision shown in Fig. 7, although all the pa- rameters are the same, the outcome in completely different and corresponds to MB reflection.

The anti-symmetric collisions correspond to MBs with a phase difference $\pi$, but we have also simulated collisions of MBs with the phase differences $\frac{\pi}{2}$ and $\frac{3 \pi}{2}$. For all these cases the colliding MBs are reflected.

A conclusion is that in a Morse chain with harmonic inter-site potential, the trapping phenomenon only appears for symmetric collisions (OS and IS).

The possible outcomes in Morse chains corresponding to the four type of collisions are summarized in Table 1.

\begin{tabular}{|c|c|c|c|}
\hline \multicolumn{4}{|c|}{ Morse chain } \\
\hline $\begin{array}{c}\text { OS } \\
\text { sym. coll. }\end{array}$ & $\begin{array}{c}\text { IS } \\
\text { sym. coll. }\end{array}$ & $\begin{array}{c}\text { OS } \\
\text { anty-sym. coll. }\end{array}$ & $\begin{array}{c}\text { IS } \\
\text { anty-sym. coll. }\end{array}$ \\
\hline $\mathrm{T}+\mathrm{MBg}$ & $\mathrm{T}+\mathrm{MBg}$ & $\mathrm{MBr}$ & $\mathrm{MBr}$ \\
\hline
\end{tabular}

Table 1. T: stationary trapped breather; $\mathrm{MBg}$ : moving breather generation; MBr: moving breather reflection. 

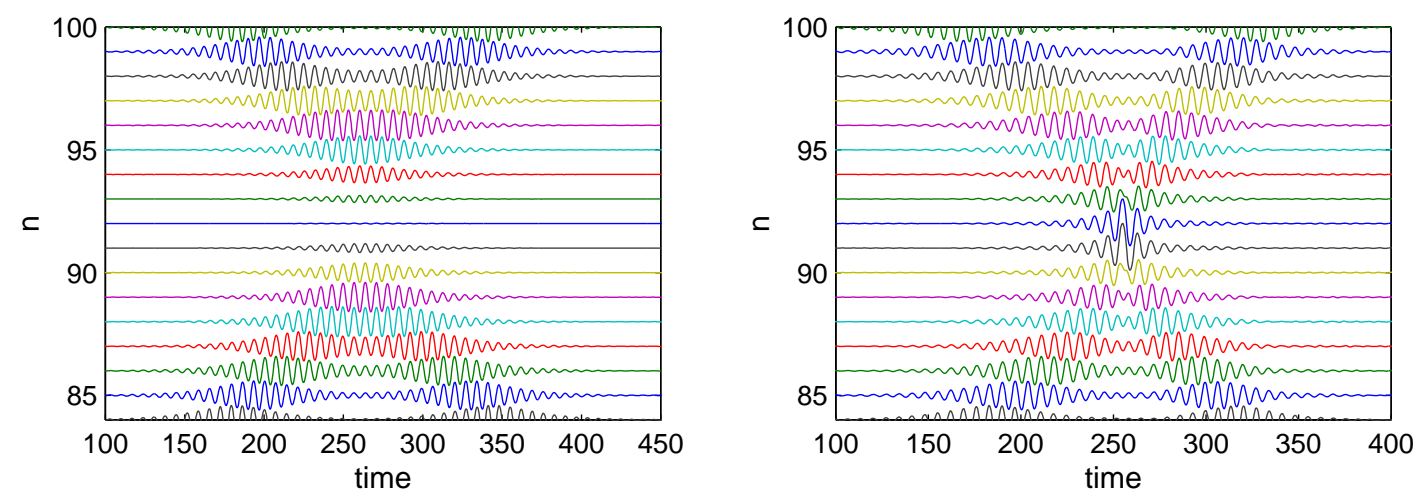

Fig. 10. (left) Zoom of the collision region corresponding to a MB reflection. (right) Zoom of the collision region corresponding to a MB generation without trapping.

\section{Collision simulations in sine-Gordon chains}

The sine-Gordon potential is also soft, and we have checked that a chain with $\varepsilon=0.50$ supports stable DBs with $\omega_{\mathrm{b}}=0.9$. Although we have analyzed the cases with different values of $\alpha$, we present the results obtained with $\alpha=0.138$ and different values of $N_{c}$. This is a representative case and permits a comparison with the collision phenomena in Morse chains.

\subsubsection{Symmetric collisions}

In the case of OS symmetric collisions, the outcome shows an oscillating trapped breather that decays very slowly to a static breather. This type of movement can be appreciated in Fig. 12 that represents a collision with $j j=20$. The central particle separating initially the MBs acts as a nucleator for the trapping of energy, and the phenomenon of MB generation does not appear when $j j$ varies. In spite of the symmetry of the equations of motion and the initial conditions of the system, we have observed a symmetry breaking in the long time regime due to numerical noise (it is not shown in the figure). Similar phenomena have been found in simulations with symmetric breather collisions in lattices with saturable nonlinearity [26].

The outcomes are different for IS symmetric collisions. The collisions can generate two MBs traveling with opposite velocities. These velocities are very sensitive with respect to $j j$, and occasionally a transient phenomenon of trapping appears which ends with the generation of two MBs. For small enough $\alpha$ values an oscillating trapped breather, as in the case of OS symmetric collisions, can occasionally appear. Fig. 13 illustrates three IS collisions with the same parameters of Fig. 12 and three consecutive $j j$ values. The $j j$ value of Fig. 13 (central) is the same of the one of Fig. 12 and the outcomes are drastically different.

There is a difference with respect to Morse chains: In Morse chains MB generation without trapping can appears for OS symmetric collisions but in sine-Gordon chains it appears for IS symmetric collisions.

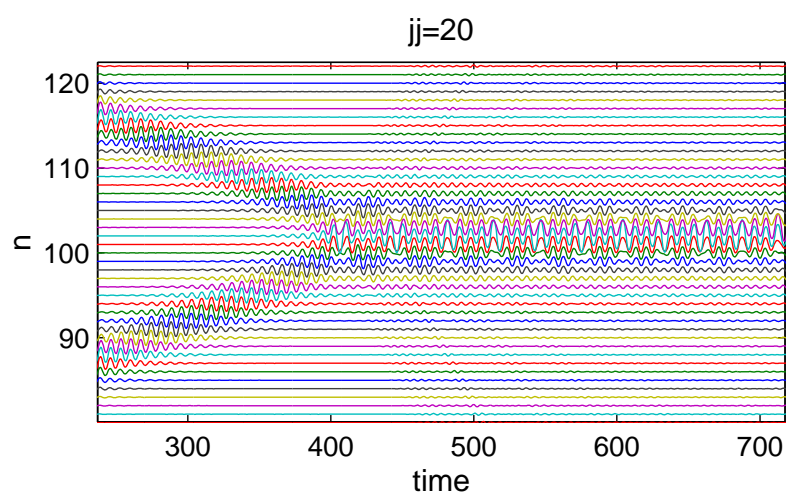

Fig. 12. sine-Gordon chain. Displacements versus time of an OS symmetric collision with $\alpha=0.138$. Coupling parameter $\varepsilon=0.50$ and breather frequency $\omega_{\mathrm{b}}=0.9$.

\subsubsection{Anti-symmetric collisions}

The results are similar to that obtained for Morse chains. The collisions always generate two reflected MBs with the same velocities that the incidents'.

The possible outcomes in Morse chains are summarized in Table 2.

\begin{tabular}{||c|c|c|c||}
\hline \hline \multicolumn{4}{|c||}{ sine-Gordon chain } \\
\hline $\begin{array}{c}\text { OS } \\
\text { sym. coll. }\end{array}$ & $\begin{array}{c}\text { IS } \\
\text { sym. coll. }\end{array}$ & $\begin{array}{c}\text { OS } \\
\text { anty-sym. coll. }\end{array}$ & $\begin{array}{c}\text { IS } \\
\text { anty-sym. coll. }\end{array}$ \\
$\mathrm{T}$ & $\mathrm{T}$ or MBg & $\mathrm{MBr}$ & $\mathrm{MBr}$ \\
\hline \hline
\end{tabular}

Table 2. T: stationary trapped breather; MBg: moving breather generation; MBr: moving breather reflection. 

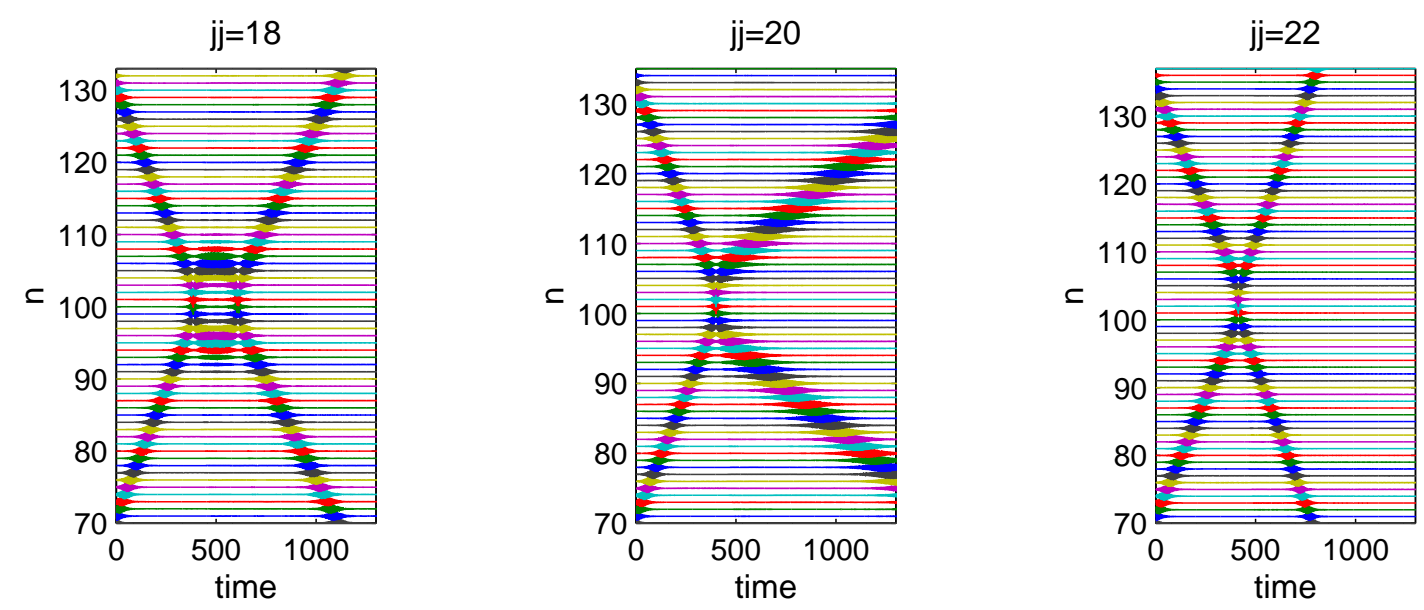

Fig. 13. sine-Gordon chain. Displacements versus time of three IS symmetric collisions with $\alpha=0.138$. Coupling parameter $\varepsilon=0.50$ and breather frequency $\omega_{\mathrm{b}}=0.9$.

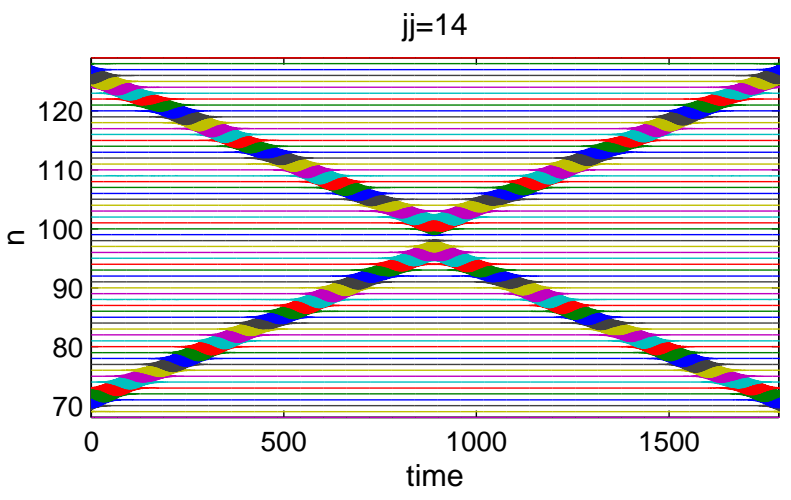

Fig. 14. $\phi^{4}$ chain. Displacements versus time of an IS symmetric collision with $j j=14$ and $\alpha=0.138$. Coupling parameter $\varepsilon=0.54$ and breather frequency $\omega_{\mathrm{b}}=3$

\section{Collision simulations in $\phi^{4}$ chains}

The $\phi^{4}$ on-site potential is hard, and we have checked that a chain with $\varepsilon=0.54$ supports stable DBs with $\omega_{\mathrm{b}}=3$. We present the results obtained with the value $\alpha=0.138$ and different values of $N_{c}$, that permit a comparison with the results obtained for the other two chains.

\subsubsection{Symmetric collisions}

In the case of OS symmetric collisions, the outcome is always $\mathrm{MB}$ generation with a trapped breather. A symmetry breaking due to numerical noise is also observed.

In the case of IS symmetric collisions a fundamental difference with respect to the Morse and sine-Gordon chains appears. Whereas in Morse and sine-Gordon chains the breather reflection appears only for anti-symmetric collisions (OS and IS), in the $\phi^{4}$ chain MB reflection appears for symmetric collisions (IS). Fig. 14 shows a symmetric IS collision for $j j=14$ and $\alpha=0.138$.

\subsubsection{Anti-symmetric collisions}

Finally, another fundamental difference appears in the case of IS anti-symmetric collisions. Both in Morse chains and in sine-Gordon chains the MBs are always reflected, but in $\phi^{4}$ chains IS anti-symmetric collisions result in MB generation without trapping. The velocities of the new MBs change when $j j$ changes. In some cases there is a broken symmetry, as in the case of $j j=14$ in Fig. 15.

The possible outcomes in $\phi^{4}$ chains are summarized in Table 3 .

\begin{tabular}{||c|c|c|c||}
\hline \hline \multicolumn{3}{|c||}{$\phi^{4}$ chain } \\
sym. coll. & IS & OS & IS \\
sym. coll. & anty-sym. coll. & anty-sym. coll. \\
T+MBg & $\mathrm{MBr}$ & $\mathrm{MBr}$ & $\mathrm{MBg}$ \\
\hline \hline
\end{tabular}

Table 3. T: stationary trapped breather; $\mathrm{MBg}$ : moving breather generation; MBr: moving breather reflection.

\section{Breather stability and trapping}

At present, it is impossible to give a theoretical explanation for the phenomena mentioned in the previous sections. Nobody has succeed in obtaining MBs as exact solutions of the dynamical equations, and the study of the collisions only can can be carried out by means of numerical simulations.

The simulations permit some estimative calculations relative to the collisions resulting in breather generation with trapping. They make possible to get some insights into the trapping phenomena based on the stability properties of the stationary breathers. 

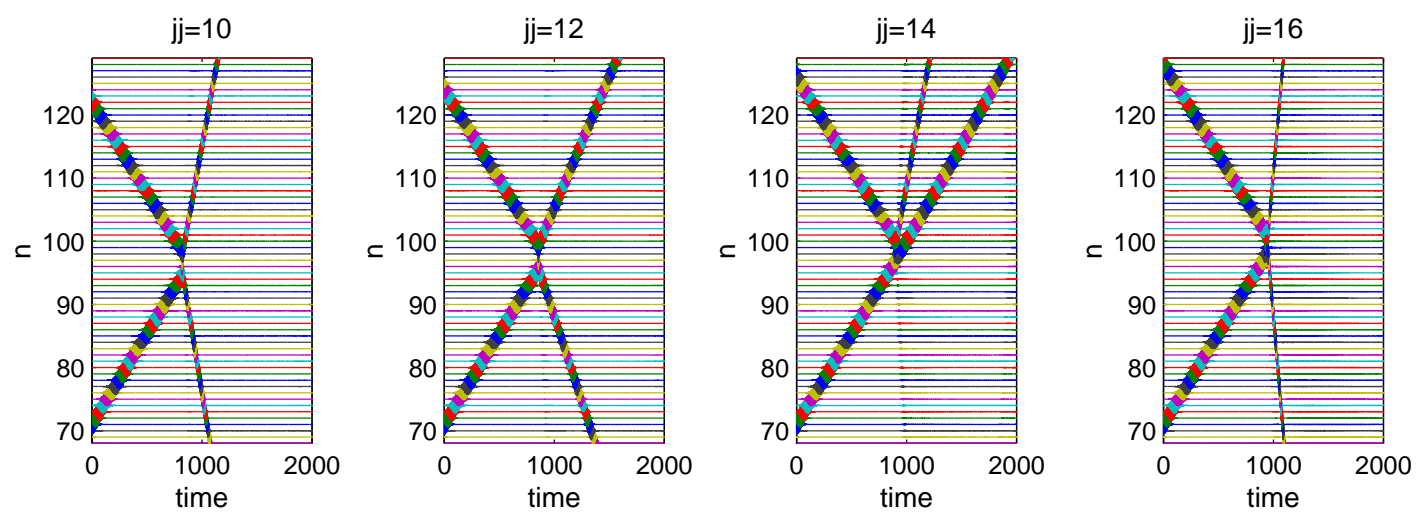

Fig. 15. $\phi^{4}$ chain. Displacements versus time of four IS anti-symmetric collisions with $j j \in[10,16]$. and $\alpha=0.138$. Coupling parameter $\varepsilon=0.54$ and breather frequency $\omega_{\mathrm{b}}=3$

Consider a symmetric collision resulting in breather generation with trapping, as shown in Fig. 2 (a). The internal and kinetic energies of the breathers involved and the energy emitted through phonon radiation are related by

$$
2 U_{\text {in }}+2 K_{\text {in }}=U_{\text {trap }}+2 U_{\text {out }}+2 K_{\text {out }}+U_{\text {ph }},
$$

where $U_{\text {in }}$ and $K_{\text {in }}$ represent the internal and kinetic energies of each one of the incident breathers; $U_{\text {out }}$ and $K_{\text {out }}$ represent the internal and kinetic energies of each one of the emerging breathers; $U_{\text {trap }}$ and $U_{\mathrm{ph}}$ represent, the internal energy of the trapped breather and the energy emitted through phonon radiation during the collision, respectively.

The on-site potential of a Klein-Gordon chain is soft and, for this type of potential, an increase of the breather frequency corresponds to a decrease of the internal energy of the breather. The energy of the stationary breather versus $\omega_{\mathrm{b}}$, with $\varepsilon=0.32$, is shown in Fig. ??(left). The frequency of a trapped breather is always different from the frequency of the incoming breathers, as the Fourier spectra show. The generation of a trapped breather requires an amount of energy, $U_{\text {trap }}$, approximately equal to the energy of a stationary breather with the same frequency. Then, if the trapped breather has a frequency lower than the frequency of the incoming breathers, $U_{\text {trap }}>U_{\text {in }}$, and viceversa.

The sum of the internal and kinetic energies of a MB depends on $\alpha, \varepsilon$ and $\omega_{\mathrm{b}}$. Fig. 16(right) shows the dependence of this sum with respect to $\alpha$ for MBs with $\varepsilon=0.32$ and $\omega_{\mathrm{b}}=0.8$ (top), or $\omega_{\mathrm{b}}=0.95$ (bottom).

Considering the collision shown in Fig. 2 (a), the frequencies of the trapped and emerging breathers are $\omega_{\text {trap }}=$ 0.77 and $\omega_{\mathrm{e}}=0.90$, respectively. The following approximate results hold $2 U_{\text {in }}+2 K_{\text {in }}=2.6,2 U_{\text {out }}+2 K_{\text {out }}=1$, $U_{\text {trap }}=1.5$ and $U_{\mathrm{ph}}=0.1$, which means that about $3.8 \%$ of the incident energy is lost as phonon radiation.

As we have shown, when two MBs collide, the excited region emits phonon radiation and the oscillators have a small frequency shift. For symmetric collisions, this shift depends on $\alpha, \varepsilon$ and $\omega_{\mathrm{b}}$. If a stationary breather with this new frequency is stable, a trapped breather can appear and the remaining energy is emitted as two new MBs traveling with opposite directions (see Fig. 2 (a), (b)) or as additional phonon radiation. However, if for the new frequency the stationary breather is unstable, there is no trapping at all and two new MBs appear traveling with opposite directions (see Fig. 2(c)).

The stability of a breather can be studied by means of its Floquet eigenvalues. Considering a breather solution $\left\{u_{n}(t)\right\}$ with period $T$, if $\xi(t)$ and $\pi(t)$ represent a perturbation of the positions and velocities with respect to $\left\{u_{n}(t)\right\}$, the Floquet matrix $\mathcal{F}$ is defined as

$$
\left[\begin{array}{l}
\xi(T) \\
\pi(T)
\end{array}\right]=\mathcal{F}\left[\begin{array}{l}
\xi(0) \\
\pi(0)
\end{array}\right]
$$

The Floquet matrix can be obtained by numerically integrating the perturbation equations:

$\ddot{\xi}_{n}(t)+V^{\prime \prime}\left(u_{n}(t)\right) \cdot \xi_{n}(t)+\varepsilon\left(2 \xi_{n}(t)-\xi_{n+1}(t)-\xi_{n-1}(t)\right)=0$.

The $2 N$ eigenvalues of $\mathcal{F},\left\{\lambda_{i}\right\}$, are called the Floquet multipliers. They can be expressed as $\lambda_{i}=\exp \left(\mathrm{i} \theta_{i}\right)$, where the complex numbers $\left\{\theta_{i}\right\}$ are called the Floquet arguments.

The perturbation equations are real and symplectic, which implies [6] that if $\lambda_{i}$ is a multiplier, the complex conjugate $\lambda_{i}^{*}$, the inverse $1 / \lambda_{i}$ and $1 / \lambda_{i}^{*}$ are also multipliers. A perturbation parallel to $\dot{u}_{n}$ is also solution to the perturbation equations and it is called the phase mode because it represents a change in the phase. As it is periodic, its multiplier has modulus 1 and, therefore, there is always a double 1 among the Floquet multipliers.

A breather is stable if every multiplier satisfy $\left|\lambda_{i}\right| \leq 1$, but if, for some $i,\left|\lambda_{i}\right|<1$, then $\left|1 / \lambda_{i}\right|>1$. Therefore the stability condition is that every eigenvalue has modulus 1 (i.e., it belongs to the unit circle), or, equivalently, every Floquet argument is a real number.

If $\left\{u_{n}(t)\right\}$ corresponds to a stable breather with frequency $\omega_{\mathrm{b}}$, all the multipliers belong to the unit circle. If the frequency changes, the multipliers move along the circle, except the double 1 corresponding to the phase mode. 

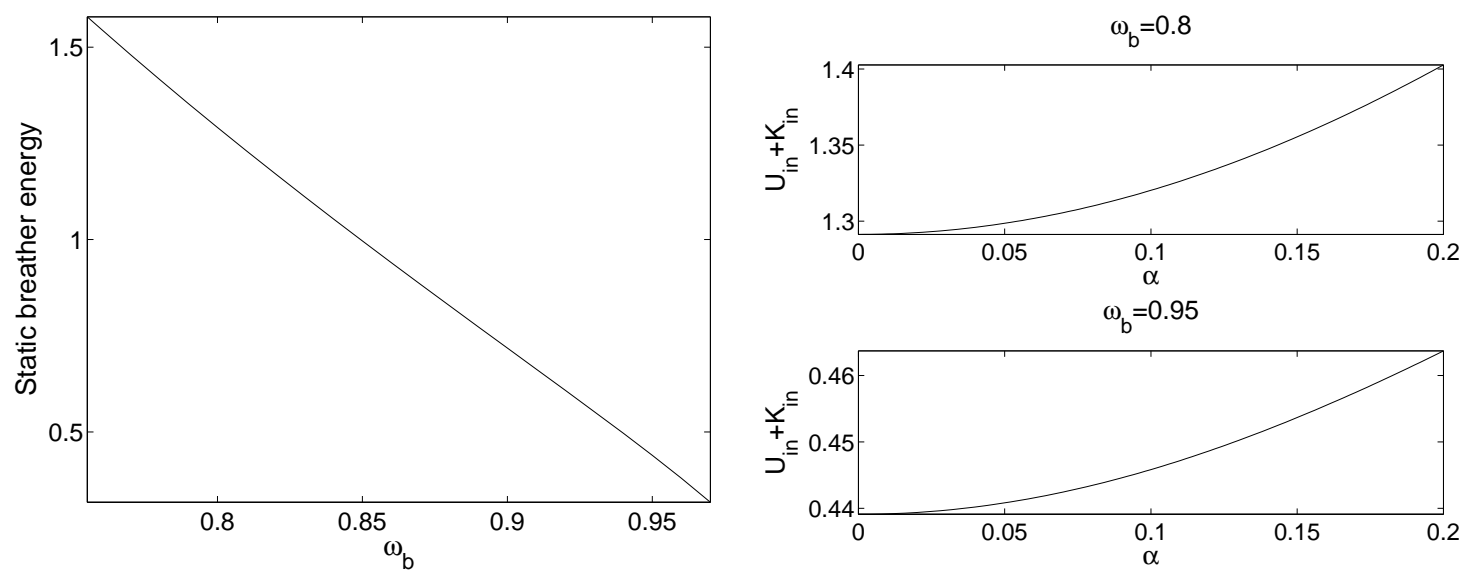

Fig. 16. (Left) Dependence of the energy of a stationary breather with respect to $\omega_{\mathrm{b}}$ with $\varepsilon=0.32$. The maximum value of the energy is $\tilde{E}=1.5798$ and corresponds to $\omega_{\mathrm{b}}=\sqrt{0.25+\varepsilon}=0.7550$ (resonance of $2 \omega_{\mathrm{b}}$ with the upper edge of the phonon band). (Right) Sum of the internal and kinetic energies versus $\alpha$ for breathers with $\varepsilon=0.32$ and $\omega_{\mathrm{b}}=0.8$ (top), or $\omega_{\mathrm{b}}=0.95$ (bottom).

The breather becomes unstable when the multipliers leave the circle and a stability bifurcation takes place. There are only three different bifurcation types:

a) Harmonic bifurcation: two multipliers coincide at 1 and leave the unit circle as real positive numbers, one smaller and the other larger than 1.

b) Subharmonic bifurcation: two multipliers coincide at -1 and leave the unit circle as real negative numbers, one smaller and the other larger than -1 .

c) Oscillatory bifurcation: two complex eigenvalues collide and leave the unit circle as complex numbers (and their complex conjugates).

A further constraint for the appearance of a bifurcation is that the Krein signatures of the multipliers that are going to leave the unit circle must have different signs [6]. The Krein signature $\kappa\left(\lambda_{i}\right)$ of a complex multiplier $\lambda_{i}$ with eigenvector $\left[\left\{\xi_{n}^{i}\right\},\left\{\pi_{n}^{i}\right\}\right]$ is defined as:

$$
\kappa\left(\lambda_{i}\right)=\operatorname{sign}\left(\sum_{n} \mathrm{i}\left[\xi_{n}^{i}(t) \pi_{n}^{i *}(t)-\xi_{n}^{i *}(t) \pi_{n}^{i}(t)\right]\right),
$$

which does not change with time due to the symplectiness of Eqs. (9). The Krein signature $\kappa\left(\lambda_{i}\right)$ of a real multiplier is zero.

If $u_{n}=0, \forall n$, the solutions of Eqs. (9) are the phonons given by $\xi_{n}^{ \pm q}=\exp \left[ \pm \mathrm{i}\left(\omega_{\mathrm{ph}}(q) t-q n\right)\right]$ with frequencies $\omega_{\mathrm{ph}}(q)=\left[\omega_{0}^{2}+4 \varepsilon \sin ^{2}(q / 2)\right]^{1 / 2}$ and wave numbers $q=$ $2 \pi m / N, m=0, \ldots, N-1$. The values of these solutions and their derivatives at $t=0$, i.e., $\left[\left\{\xi_{n}^{ \pm}(0)\right\},\left\{\dot{\xi}_{n}^{ \pm}(0)\right\}\right]$ are eigenvectors of the Floquet matrix, with multipliers $\lambda( \pm q)=\exp \left( \pm \mathrm{i} \omega_{\mathrm{ph}}(q) T\right)=\exp \left( \pm \mathrm{i} 2 \pi \omega_{\mathrm{ph}}(q) / \omega_{\mathrm{b}}\right)$, arguments $\theta( \pm q)= \pm 2 \pi \omega_{\mathrm{ph}}(q) / \omega_{\mathrm{b}}(\bmod 2 \pi)$, and Krein signatures $\kappa( \pm q)= \pm 1$. We will call them, for short, the phonon multipliers and phonon arguments.

If $\left\{u_{n}\right\}$ corresponds to a breather solution, most of the Floquet multipliers will be almost equal to the phonon ones, and more so, the larger the system. They will form two phonon bands within the unit circle, one with Floquet arguments between $2 \pi \omega_{0} / \omega_{\mathrm{b}}$ and $2 \pi\left[\omega_{0}^{2}+4 \varepsilon \sin ^{2}(q / 2)\right]^{1 / 2} / \omega_{\mathrm{b}}$ with Krein signature +1 , and the corresponding complex conjugate band with Krein signature -1 . For example, for $\varepsilon=0.19$ and $\omega_{\mathrm{b}}=0.8$, the phonon band " + " (with positive Krein signature) extends from $90^{\circ}$ to $237^{\circ}$, the phonon band "-" extends from $-90^{\circ}$ to $-237^{\circ}$. That is, the two phonon bands overlap and there are arguments with different Krein signature very close one to each other. Note that we have used in our simulations around 100 or 200 oscillators, therefore, there are nearly 100 or 200 Floquet arguments in each phonon band.

If $\omega_{\mathrm{b}}$ changes, the phonons Floquet arguments move along the circle and many with different Krein signatures will cross, bringing about the possibility of instabilities. If we calculate numerically the Floquet arguments as a function of $\omega_{\mathrm{b}}$, we can observe that usually they leave the unit circle (i.e. the breather becomes unstable) and come back again inside it. There are very many frequency islands of stability and instability. Hence the extreme sensitivity of the outcome of the collision to the initial conditions. Moreover, not only the stability of the candidate to trapped breather changes but also the eigenvector corresponding to that instability and, as a consequence, the particular result of the non-trapping collision. At present, we can not predict the small frequency shifts resulting of a symmetric collision, and therefore, its outcome.

\section{Conclusions}

In this paper, we have studied, by means of numerical simulations, the collisions of moving discrete breathers in three different Klein-Gordon chains of oscillators. In the first chain the on-site potential is the asymmetric and soft Morse potential and the inter-site potential is harmonic. In the second chain the on-site potential is the symmetric and soft sine-Gordon potential and the inter-site potential is 
harmonic. Finally, in the third chain the on-site potential is the symmetric and hard $\phi^{4}$ potential and the inter-site potential is non-harmonic.

A collision simulation begins generating two identical moving breathers traveling with opposite velocities. We have considered: symmetric collisions, when the colliding moving breathers are vibrating in phase; anti-symmetric collisions, when the colliding moving breathers are vibrating in anti-phase. For both cases we have considered onsite collisions and inter-site collisions.

The simulations show that the collision properties of the three chains are different. The principal observed phenomena are: breather generation with trapping, with the appearance of two new moving breathers with opposite velocities and a stationary breather trapped at the collision region; breather generation without trapping, with the appearance of new moving breathers with opposite velocities; breather trapping at the collision region, without the appearance of new moving breathers; and breather reflection.

For each Klein-Gordon chain, the collision outcomes depend on the lattice parameters, the frequency of the perturbed stationary breathers, the internal structure of the moving breathers and the number of particles that initially separates the stationary breathers when they are perturbed.

At present, nobody has succeed in obtaining MBs as exact solutions of the dynamical equations, and the study of the collisions only can be carried out by means of numerical simulations. Nevertheless, our simulations permit some estimative calculations relative to the collisions resulting in breather generation with trapping. They make possible to get some insights into the trapping phenomena based on the stability properties of the stationary breathers.

Apart of the dependence on the lattice parameters, we have observed that in many cases the outcomes are very sensitive with respect to the internal structure of the moving breathers, and the exact number of particles that initially separates the stationary breathers when they are perturbed.

\section{References}

1. Focus issue edited by $\mathrm{T}$ Dauxois, RS Mackay and GP Tsironis, Condensed Matter, Dynamical Systems and Biophysics, Physica D 216, (2006) 1

2. Focus issue edited by Yu S Kivshar and S Flach, Nonlinear localized modes: physics and applications, Chaos 13, (2003) 586

3. Focus issue edited by S Flach and RS Mackay, Localization in nonlinear lattices, Physica D 119, (1999) 1

4. S Flach and CR Willis, Phys. Rep. 295, (1998) 181

5. RS MacKay and S Aubry, Nonlinearity 7, (1994) 1623

6. S Aubry, Physica D 103, (1997) 201

7. AJ Sievers and S Takeno, Phys. Rev. Lett. 61, (1988) 970

8. J. Cuevas B. Sánchez-Rey, G. James and J.F.R. Archilla, Phys. Rev. B 70, (2004) 014301

9. K.Ø. Rasmussen P.G. Kevrekidis and A.R. Bishop, Int. J. Mod. Phys. B 15, (2001) 2833
10. Ding Chen, S Aubry, and GP Tsironis, Phys. Rev. Lett. 77, (1996) 4776

11. S Aubry and T Cretegny, Physica D 119, (1998) 34

12. J Cuevas, JFR Archilla, Yu B Gaididei, and FR Romero, Physica D 163, (2002) 106

13. J Cuevas, F Palmero, JFR Archilla, and FR Romero, Phys. Lett. A 299, (2002) 221

14. J Cuevas, F Palmero, JFR Archilla, and FR Romero, J. Phys. A: Math. and Gen. 35, (2002) 10519

15. M Peyrard and AR Bishop, Phys. Rev. Lett. 62, (1989) 2755

16. T Dauxois, M Peyrard, and CR Willis, Physica D 57, (1992) 267

17. Y. Doi, Phys. Rev. E 68, (2003) 066608

18. T Dauxois and M Peyrard, Phys. Rev. Lett. 70, (1993) 3935

19. K Forinash, M Peyrard, and BA Malomed, Phys. Rev. E 49, (1994) 3400

20. K Forinash, T Cretegny, and M Peyrard, Phys. Rev. E 55, (1997) 4740

21. M. Meister and L.M. Floría, Eur. Phys. J. B 37, (2004) 213

22. D. Cai, A.R. Bishop, and N. Gronbech-Jensen, Phys. Rev. E 56, (1997) 7246

23. S.V. Dmitriev, P.G. Kevrekidis, B.A. Malomed, and D.J. Frantzeskakis, Phys. Rev. E 68, (2003)056603

24. I.E. Papacharalampous, P.G. Kevrekidis, B.A. Malomed, and D.J. Frantzeskakis, Phys. Rev. E 68, (2003) 046604

25. J. Cuevas and J.C. Eilbeck, Phys. Lett. A 358, (2006) 15

26. A. Maluckov, L. Hadzievski, and M. Stepic, Eur.Phys.J.B $\mathbf{5 3},(2006) 333$

27. JL Marín and S Aubry, Nonlinearity 9, (1996) 1501

28. JM Sanz-Serna and MP Calvo. Numerical Hamiltonian problems (Chapman and Hall, 1994).

29. O Bang and M Peyrard, Physica D 81, (1995) 9

30. AM Morgante, M Johannson, G Kopidakis, and S Aubry, Physica D 162, (2002) 53

31. JA Sepulchre, In Localization and Energy Transfer in Nonlinear Systems (World Scientific, Singapore, 2003)

32. A Alvarez, FR Romero, J Cuevas and JFR Archilla, Phys. Lett. A 372, (2008) 1256 